\title{
BMJ Open Association of nephrolithiasis with the risk of cardiovascular diseases: a longitudinal follow-up study using a national health screening cohort
}

\author{
So Young Kim, ${ }^{1}$ Woo Jin Bang, ${ }^{2}$ Chanyang Min, ${ }^{3}$ Hyo Geun Choi (D) ${ }^{4}$
}

To cite: Kim SY, Bang WJ, Min C, et al. Association of nephrolithiasis with the risk of cardiovascular diseases: a longitudinal follow-up study using a national health screening cohort. BMJ Open 2020;10:e040034. doi:10.1136/ bmjopen-2020-040034

- Prepublication history and additional material for this paper are available online. To view these files, please visit the journal online (http://dx.doi. org/10.1136/bmjopen-2020040034).

SYK and WJB contributed equally.

Received 03 May 2020 Revised 15 September 2020 Accepted 21 October 2020
D) Check for updates

(C) Author(s) (or their employer(s)) 2020. Re-use permitted under CC BY-NC. No commercial re-use. See rights and permissions. Published by BMJ.

For numbered affiliations see end of article.

Correspondence to

Dr Hyo Geun Choi;

pupen@naver.com

\section{ABSTRACT}

Objectives The aim of this study was to explore the associations of stroke and ischaemic heart disease in patients with nephrolithiasis.

Design A longitudinal follow-up study.

Setting Data from the Korean National Health Insurance Service-Health Screening Cohort (2002-2013) were retrieved to identify the occurrence of nephrolithiasis. Participants and interventions In total, 19103 patients with nephrolithiasis were matched at a 1:4 ratio with control participants for age, sex, income and region of residence.

Primary and secondary outcome measures The occurrence of stroke and ischaemic heart disease was analysed in both patients with nephrolithiasis and control participants. The primary outcome was HRs of stroke and ischaemic heart disease in a stratified Cox proportional hazards model. Smoking, alcohol consumption, obesity and Charlson Comorbidity Index were adjusted for as covariates. Subgroup analyses according to age and sex were also performed.

Results Eight per cent (1615/19 103) of patients with nephrolithiasis and $7.2 \%$ (5476/76 412) of control participants had stroke. Nine per cent (1879/19 103) of patients with nephrolithiasis and $7.7 \%$ (5895/76 412) of control participants had ischaemic heart disease. Patients with nephrolithiasis had risks of stroke and ischaemic heart disease that were 1.18 times $(95 \% \mathrm{Cl}=1.11$ to 1.24$)$ and 1.24 times $(95 \% \mathrm{Cl}=1.18$ to 1.31$)$ those of the control participants, respectively. The age and sex subgroups showed consistent results.

Conclusions Nephrolithiasis was associated with increased risks of stroke and ischaemic heart disease.

\section{INTRODUCTION}

Nephrolithiasis is a common urinary tract disease. Approximately $4.2 \%-10.1 \%$ of the worldwide population and $3.5 \%$ of the Korean population have nephrolithiasis. $^{1-3}$ The prevalence of nephrolithiasis is increasing worldwide. ${ }^{4}$ The increasing prevalence of obesity has been suspected to promote the formation of nephrolithiasis. ${ }^{5}$ Acute renal colic due to blockage of the ureter is an acute urinary manifestation of
Strengths and limitations of this study

- This study adds to previous findings by analysing a large cohort. The large study population provided many control participants matched for age, sex, income and region of residence.

- The lifestyle factors of obesity, smoking status and alcohol consumption and the additional factors of Charlson Comorbidity Index, total cholesterol and fasting blood glucose were adjusted for to minimise confounding by these covariates.

- Because these data were based on medical claim codes, subclinical or untreated patients might have been missed in this study.

nephrolithiasis. In addition, nephrolithiasis can be associated with systemic comorbidities, including metabolic syndrome. ${ }^{6}$ For decades, a growing number of epidemiological studies have suggested the association of nephrolithiasis with systemic comorbidities, such as diabetes and hypertension. ${ }^{78}$ Because these comorbidities are predisposing conditions for cardiovascular disorders, researchers have also explored the associations between nephrolithiasis and cardiovascular disorders. ${ }^{9-11}$

Previous studies have reported an association between nephrolithiasis and stroke. ${ }^{910}$ However, to our knowledge, neither our prior study nor most other published studies have considered the impacts of lifestyle factors, including obesity, smoking and alcohol consumption, on the association between nephrolithiasis and stroke. Because renal stone formation, as well as cardiovascular disease, has been suggested to be related to obesity and smoking, the possible confounding effects of these covariates should be controlled to elucidate the true association between nephrolithiasis and cardiovascular diseases. ${ }^{12} 13$ In addition, because cerebrovascular disease (stroke) and cardiovascular disease (ischaemic heart disease) are 
associated with each other, these vascular disorders need to be independently considered regarding their relationship with nephrolithiasis.

We hypothesised that nephrolithiasis might increase the risks of both stroke and ischaemic heart disease, probably due to their shared pathophysiology. This study is an improvement on our previous study on the association between nephrolithiasis and stroke due to the inclusion of ischaemic heart disease. In addition, potential confounders, including obesity, smoking and alcohol consumption, were considered. The risks of stroke and ischaemic heart disease were analysed by adjusting for medical histories using the Charlson Comorbidity Index (CCI) and lifestyle factors, including obesity, smoking and alcohol consumption. Because prior studies indicated that there might be sex-based differences in the association between nephrolithiasis and cardiovascular disease,${ }^{10}$ subgroup analyses were conducted.

\section{MATERIALS AND METHODS Study population}

A detailed description of the Korean National Health Insurance Service-Health Screening Cohort (NHIS-NSC) data is available elsewhere. ${ }^{14}$

\section{Definition of nephrolithiasis}

Nephrolithiasis was defined if the participants were diagnosed with the International Classification of Diseases, 10th Revision (ICD-10) code N20 $\geq 2$ times, as in our previous studies. ${ }^{9} 15$

\section{Definition of stroke and ischaemic heart disease}

Stroke and ischaemic heart disease were identified based on ICD-10 codes (I60-I69 for stroke and I20-I25 for ischaemic heart disease), as in our previous study. ${ }^{9}$

\section{Participant selection}

Patients with ephrolithiasis ( $\mathrm{n}=22003)$ were selected from 514866 participants with 497931549 medical claim codes. The control group included participants who were never diagnosed with nephrolithiasis from 2002 to 2013 $(n=492863)$. Patients with nephrolithiasis were matched at a 1:4 ratio with the control participants for age, sex, income and region of residence. To minimise selection bias, the control participants were selected with random number generation. The index date of each patient with nephrolithiasis was considered the date of the first diagnosis of nephrolithiasis. The index date of the control participants was considered the index date of their matched patient with nephrolithiasis . Therefore, each matched patient with nephrolithiasis and their respective control participants had the same index date. Nine patients with nephrolithiasis who had previous stroke or ischaemic heart disease before the index date were excluded. Control participants who had previous stroke or ischaemic heart disease before the index date were also excluded. Among the control participants, 404887 were excluded during the matching procedure. Finally, 19103 patients with nephrolithiasis were 1:4 matched with 76412 control participants (figure 1). The patients with nephrolithiasis who visited the emergency department or were hospitalised were classified as having severe nephrolithiasis, whereas others were classified as having mild to moderate nephrolithiasis.

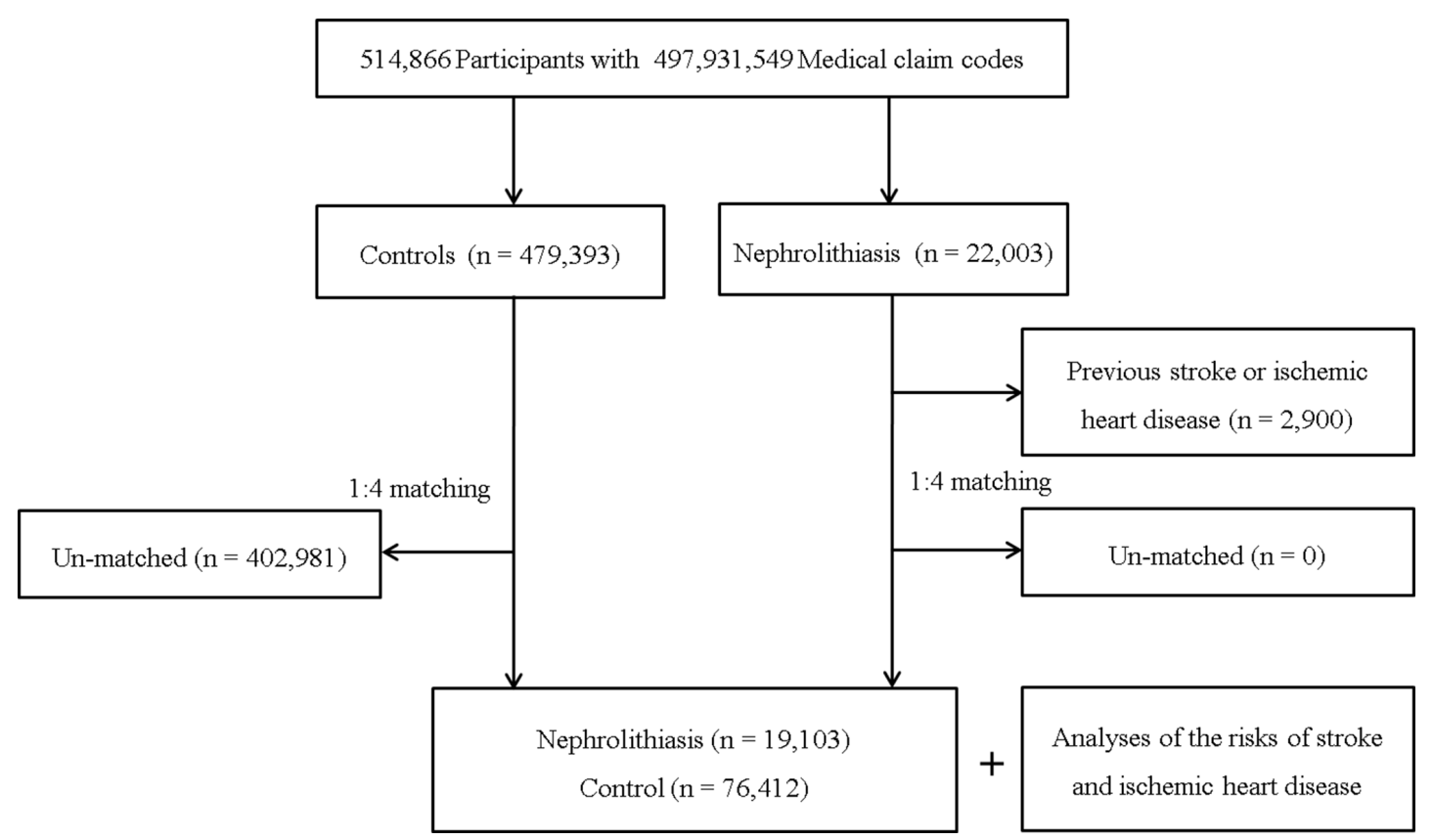

Figure 1 A schematic illustration of the participant selection process that was used in this study. Of a total of 514866 participants, 21994 patients with nephrolithiasis were matched with 87976 control participants for age, sex, income and region of residence. 


\section{Covariates}

Patients were divided into age groups with 5-year intervals: $40-44,45-49,50-54, \ldots$ and $85+$ years. A total of 10 age groups were specified. There were five income classes (classes 1 (lowest income) to 5 (highest income)). The region of residence was categorised as urban (Seoul, Busan, Daegu, Incheon, Gwangju, Daejeon and Ulsan) or rural (Gyeonggi, Gangwon, Chungcheongbuk, Chungcheongnam, Jeollabuk, Jeollanam, Gyeongsangbuk, Gyeongsangnam and Jeju).

Tobacco smoking was categorised based on the participant's current smoking status (non-smoker, past smoker or current smoker). Alcohol consumption was categorised based on the frequency of alcohol consumption (non-drinker, 1-3 times a month and $\geq 1$ time a week) because previous studies indicated a positive association of nephrolithiasis with binge drinking $^{16}$ and a negative association with moderate alcohol consumption. ${ }^{17}$ Obesity was determined according to the body mass index (BMI, $\left.\mathrm{kg} / \mathrm{m}^{2}\right)$. Missing BMI variables were replaced by the mean $\mathrm{BMI}$ of the final selected participants. BMI was categorised as $<18.5$ (underweight), $\geq 18.5$ to $<23$ (normal), $\geq 23$ to $<25$ (overweight), $\geq 25$ to $<30$ (obese I) or $\geq 30$ (obese II) based on the Asia-Pacific criteria produced by the Western Pacific Regional Office 2000. ${ }^{18}$ Serum levels of total cholesterol $(\mathrm{mg} / \mathrm{dL})$ and fasting glucose $(\mathrm{mg} / \mathrm{dL})$ were included as continuous variables.

The CCI has been widely used to measure the disease burden based on 17 comorbidities. A score was calculated for each participant depending on the number and severity of diseases. The CCI was measured as a continuous variable (0 (no comorbidities) to 29 (multiple comorbidities)). ${ }^{19} 20$ The scores excluding cerebrovascular disease were calculated. The CCI score was evaluated as a continuous variable.

\section{Statistical analyses}

The general characteristics were compared between the nephrolithiasis and control groups with $\chi^{2}$ tests.

To analyse the HRs and $95 \%$ CIs of stroke and ischaemic heart disease in patients with nephrolithiasis compared with control participants, a stratified Cox proportional hazards model was used. In this analysis, a crude model and a model adjusted for obesity, smoking status, alcohol consumption and CCI score were generated. The analysis was stratified by matching variables such as age, sex, income and region of residence. Kaplan-Meier curves were constructed, and logrank tests were performed.

For the subgroup analyses, we stratified the participants by age and sex (aged $<60$ years and $\geq 60$ years; men and women) and severity of nephrolithiasis (mild to moderate and severe), and analysed the crude and adjusted models.

Two-tailed analyses were performed, and significance was defined as a $\mathrm{p}$ value less than 0.05. SAS V.9.4 (SAS Institute) was used for statistical analyses.

\section{Patient and public involvement}

This national cohort study used data from the NHIS-NSC. Detailed descriptions of these data are available in our previous studies. $^{21} 22$ No patients were involved in the development of the research question or the design of the study.
We have no plan to disseminate the results to the patients. Because the NHIS-NSC data are based on national health claim codes, release of the data by the researcher is illegal. All data are available from the National Health Insurance Sharing Service (NHISS) database (https://nhiss.nhis.or. $\mathrm{kr} /)$.

The NHISS allows all of these data to be used by any researcher who promises to follow the research ethics guidelines, with some associated costs. If one wants to access the data described in this article, one could download them from the website after promising to adhere to the research ethics requirements.

\section{RESULTS}

Age, sex, income and region of residence were exactly matched between the nephrolithiasis and control groups $(\mathrm{p}=1.000)$. The rates of low CCI, overweight, obesity I, obesity II and non-smoker status were higher in the nephrolithiasis group than in the control group (each $\mathrm{p}<0.05)$. The distribution of alcohol consumption and the serum level of total cholesterol were different between the nephrolithiasis group and the control group (each $\mathrm{p}<0.001)$. The prevalence of stroke and ischaemic heart disease was lower in the nephrolithiasis group than in the control group $(\mathrm{p}<0.001$; table 1$)$.

The adjusted HR of stroke in the nephrolithiasis group was $1.18(95 \% \mathrm{CI}=1.11$ to $1.24, \mathrm{p}<0.001)$ (figure $2 \mathrm{~A})$. In the subgroup analyses according to age and sex, the adjusted HRs of stroke were higher in the nephrolithiasis group than in the control group, except for in the subgroup of men aged $\geq 60$ years (table 2 ).

The adjusted HR of ischaemic heart disease in the nephrolithiasis group was $1.24(95 \% \mathrm{CI}=1.18$ to $1.31, \mathrm{p}<0.001)$ (figure 2B). In the subgroup analyses according to age and sex, the adjusted HRs of ischaemic heart disease were higher in the nephrolithiasis group than in the control group (table 3). The associations of stroke and ischaemic heart disease with nephrolithiasis remained consistent when considering a 1-year washout period (online supplemental tables S1 and S2). Patients with both mild to moderate and severe nephrolithiasis had higher odds of stroke and ischaemic heart disease (online supplemental tables S3 and S4). When the participants were stratified by smoking, alcohol consumption and obesity, the association of nephrolithiasis with stroke was consistent in all subgroups, except for in the past smoker, underweight and obese II subgroups (online supplemental tables S5 and S6).

\section{DISCUSSION}

Patients with nephrolithiasis had 1.18 times and 1.25 times higher risks of stroke and ischaemic heart disease, respectively. These increased risks of stroke and ischaemic heart disease were consistent in all age and sex subgroups, except for in men aged $\geq 60$ years, in whom there was no association between nephrolithiasis and stroke. This study added to previous findings by analysing a large cohort. The large study population provided many control participants matched for age, sex, income and region of residence. Furthermore, 
Table 1 General characteristics of participants

\begin{tabular}{lccc}
\hline \multirow{2}{*}{ Characteristics } & \multicolumn{3}{c}{ Total participants } \\
\cline { 2 - 4 } & Nephrolithiasis & \multicolumn{1}{c}{ Control } & P value \\
\hline Age (years), n (\%) & $1593(8.3)$ & $6372(8.3)$ & 1.000 \\
$40-44$ & $3659(19.2)$ & $14636(19.2)$ \\
$45-49$ & $4570(23.9)$ & $18280(23.9)$ \\
$50-54$ & $3525(18.5)$ & $14100(18.5)$ \\
$55-59$ & $2570(13.5)$ & $10280(13.5)$ \\
$60-64$ & $1709(9.0)$ & $6836(9.0)$ \\
$65-69$ & $955(5.0)$ & $3820(5.0)$ \\
$70-74$ & $402(2.1)$ & $1608(2.1)$ \\
$75-79$ & $102(0.5)$ & $408(0.5)$ \\
$80-84$ & $18(0.1)$ & $72(0.1)$ \\
$85+$ & & \\
\hline
\end{tabular}

Sex, n (\%)

\begin{tabular}{|c|c|c|c|}
\hline Male & $12303(64.4)$ & $49212(64.4)$ & \\
\hline Female & $6800(35.6)$ & 27200 (35.6) & \\
\hline Income, n (\%) & & & 1.000 \\
\hline 1 (lowest) & 2576 (13.5) & $10304(13.5)$ & \\
\hline 2 & 2269 (11.9) & 9076 (11.9) & \\
\hline 3 & $2893(15.1)$ & $11572(15.1)$ & \\
\hline 4 & $4108(21.5)$ & 16432 (21.5) & \\
\hline 5 (highest) & 7257 (38.0) & 29028 (38.0) & \\
\hline $\begin{array}{l}\text { Region of } \\
\text { residence, } n(\%)\end{array}$ & & & 1.000 \\
\hline Urban & $8667(45.4)$ & 34668 (45.4) & \\
\hline Rural & $10436(54.6)$ & $41744(54.6)$ & \\
\hline $\mathrm{CCl}$ score, n (\%) & & & $0.005^{*}$ \\
\hline 0 & 18735 (98.1) & 74671 (97.7) & \\
\hline 1 & $58(0.3)$ & $370(0.5)$ & \\
\hline 2 & $72(0.4)$ & $336(0.4)$ & \\
\hline 3 & $53(0.3)$ & $257(0.3)$ & \\
\hline$\geq 4$ & $185(1.0)$ & 778 (1.0) & \\
\hline Obesity (BMI, kg/ & & & $<0.001^{*}$ \\
\hline
\end{tabular}

$\left.\mathrm{m}^{2}\right), \mathrm{n}(\%)$

\begin{tabular}{|c|c|c|c|}
\hline $\begin{array}{l}<18.5 \\
\text { (underweight) }\end{array}$ & 267 (1.4) & 1642 (2.2) & \\
\hline $\begin{array}{l}\geq 18.5 \text { to }<23 \\
\text { (normal) }\end{array}$ & 5546 (29.0) & 27089 (35.5) & \\
\hline $\begin{array}{l}\geq 23 \text { to }<25 \\
\text { (overweight) }\end{array}$ & $5586(29.2)$ & 21246 (27.8) & \\
\hline $\begin{array}{l}\geq 25 \text { to }<30 \\
\text { (obese l) }\end{array}$ & 7069 (37.0) & 24472 (32.0) & \\
\hline$\geq 30$ (obese II) & 635 (3.3) & 1963 (2.6) & \\
\hline $\begin{array}{l}\text { Smoking status, } \\
\mathrm{n}(\%)\end{array}$ & & & $<0.001^{*}$ \\
\hline Non-smoker & $12434(65.1)$ & 48225 (63.1) & \\
\hline Past smoker & 2490 (13.0) & 9512 (12.5) & \\
\hline Current smoker & 4179 (21.9) & 18675 (24.4) & \\
\hline $\begin{array}{l}\text { Alcohol } \\
\text { consumption, n (\%) }\end{array}$ & & & $<0.001^{*}$ \\
\hline Non-drinker & $11030(57.7)$ & 40219 (52.6) & \\
\hline
\end{tabular}

Continued
Table 1 Continued

\begin{tabular}{|c|c|c|c|}
\hline \multirow{2}{*}{ Characteristics } & \multicolumn{3}{|c|}{ Total participants } \\
\hline & Nephrolithiasis & Control & $P$ value \\
\hline $\begin{array}{l}1-3 \text { times per } \\
\text { month }\end{array}$ & 2985 (15.6) & 12417 (16.3) & \\
\hline$\geq 1$ time per week & $5088(26.6)$ & 23776 (31.1) & \\
\hline $\begin{array}{l}\text { Total cholesterol } \\
\text { (mg/dL), mean (SD) }\end{array}$ & $201.8(37.2)$ & $199.4(37.3)$ & $<0.001 \dagger$ \\
\hline $\begin{array}{l}\text { Fasting blood } \\
\text { glucose } \\
\text { (mg/dL), mean (SD) }\end{array}$ & $99.8(28.8)$ & 99.5 (29.9) & 0.186 \\
\hline Stroke, n (\%) & $1615(8.5)$ & $5476(7.2)$ & $<0.001^{\star}$ \\
\hline $\begin{array}{l}\text { Ischaemic heart } \\
\text { disease, } \mathrm{n}(\%)\end{array}$ & $1879(9.8)$ & $5895(7.7)$ & $<0.001^{*}$ \\
\hline
\end{tabular}

${ }^{*} \chi^{2}$ test. Significance at $p<0.05$.

†Independent $t$ test. Significance at $p<0.05$.

$\mathrm{BMI}$, body mass index; $\mathrm{CCl}$, Charlson Comorbidity Index.

the lifestyle factors of obesity, smoking status and alcohol consumption, and medical histories were adjusted for to minimise confounding by these covariates. This study was a longitudinal follow-up study that explored the causal relationship between nephrolithiasis and stroke or ischaemic heart disease. Participants who had previous histories of stroke or ischaemic heart disease before the index date were excluded. In addition, participants who had histories of stroke or ischaemic heart disease at 1 year and 2 years after the index date were excluded from the supplementary analyses.

The metabolic perturbations in patients with nephrolithiasis, which manifest as hypercalciuria, hyperuricaemia or hyperoxaluria, could mediate the increased risk of cardiovascular plaque formation and metabolic changes associated with cardiovascular disorders. It has been suggested that the abnormal calcification process is similar in the atherosclerosis of cerebral or coronary vasculature and in the formation of nephrolithiasis. ${ }^{23}$ There is evidence of the presence of these metabolic changes in patients with nephrolithiasis; the levels of calcification inhibitors were found to be decreased in the blood and urine of patients with atherosclerosis and nephrolithiasis. ${ }^{23}$ In addition to the direct calcification process, other indirect metabolic changes in patients with nephrolithiasis might impact the risk of cardiovascular disorders. Patients with metabolic syndrome had 1.25 times $(95 \% \mathrm{CI}=1.03$ to 1.50$)$ higher odds of nephrolithiasis in a cross-sectional study. ${ }^{24}$ In an experimental animal study, a metabolic syndrome rat model with insulin resistance had an increased risk of urinary calcium stone formation. ${ }^{25}$ In a clinical study, the metabolic syndrome traits of obesity, hypertension, diabetes and dyslipidaemia were 1.78 times $(95 \% \mathrm{CI}=1.22$ to 2.66$)$ more common in patients with nephrolithiasis with recurrent or multiple stones. ${ }^{26}$ Therefore, nephrolithiasis should be considered a systemic metabolic disease rather than a local metabolic disease involving calcification. These systemic metabolic disturbances in patients with nephrolithiasis might mediate the increased risk of cardiovascular disorders. 

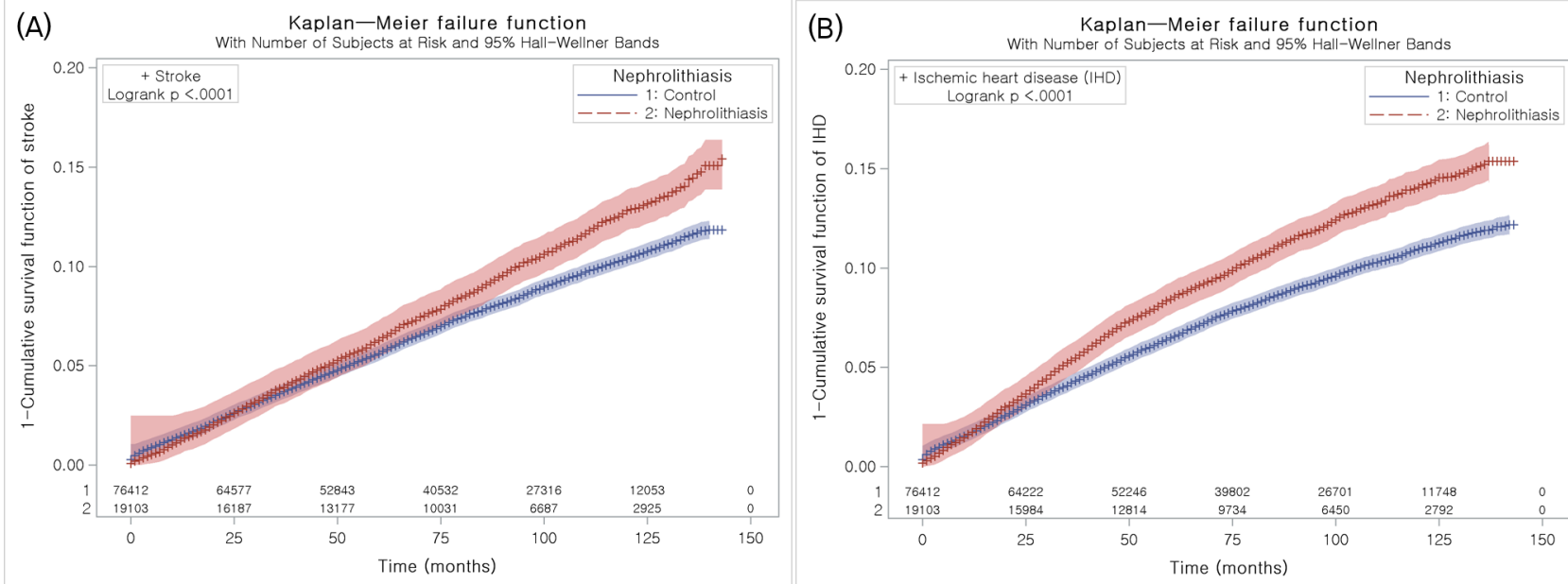

Figure 2 Kaplan-Meier survival analysis. (A) The cumulative rate of stroke was higher in the nephrolithiasis group than in the control group. (B) The cumulative rate of ischaemic heart disease was higher in the nephrolithiasis group than in the control group.

The risk of stroke was greater in patients with nephrolithiasis than in control participants in this study. Our previous study reported a 1.13 times $(95 \% \mathrm{CI}=1.06$ to 1.21$)$ greater risk of ischaemic stroke in patients with nephrolithiasis than in the control group. ${ }^{9}$ A recent meta-analysis reported that a history of nephrolithiasis was associated with a 1.23-fold $(95 \% \mathrm{CI}=1.06$ to 1.38$)$ increased relative risk of stroke. ${ }^{11}$ However, few previous studies considered smoking, obesity and alcohol consumption, and only selected comorbidities were used for adjustment. After adjusting for both lifestyle factors and medical histories, the risk of stroke was greater in patients with nephrolithiasis than in controls in this study. Moreover, the risk of ischaemic heart disease was greater in patients with nephrolithiasis than in controls in this study. A recent meta-analysis reported that the relative risk of coronary heart disease was 1.24 times $(95 \% \mathrm{CI}=1.14$ to 1.36$)$ higher in patients with nephrolithiasis. ${ }^{11}$ This figure is similar to the present HR of 1.25 (95\% CI=1.18 to 1.31 ).

The age and sex subgroup analyses indicated increased risks of stroke and ischaemic heart disease in patients with nephrolithiasis, except for in men aged $\geq 60$ years. A metaanalysis showed that the pooled HR for myocardial infarction was 1.49 (95\% CI=1.21 to 1.82$)$ in the female group, whereas there was no association between nephrolithiasis and myocardial infarction in the male group. ${ }^{10}$ This femalespecific association between nephrolithiasis and myocardial

Table 2 Crude and adjusted HRs (95\% Cls) for stroke in the nephrolithiasis and control groups

\begin{tabular}{|c|c|c|c|c|}
\hline \multirow{2}{*}{ Characteristics } & \multicolumn{4}{|c|}{ HRs for stroke } \\
\hline & Crude* & $P$ value & Adjusted $^{\star} \dagger$ & $P$ value \\
\hline \multicolumn{5}{|c|}{ Total participants $(\mathrm{n}=95515)$} \\
\hline Control & 1.00 & & 1.00 & \\
\hline \multicolumn{5}{|c|}{ Age $<60$ years, men $(n=44595)$} \\
\hline \multicolumn{5}{|c|}{ Age $<60$ years, women $(n=22140)$} \\
\hline Nephrolithiasis & $1.32(1.16$ to 1.51$)$ & $<0.001 \ddagger$ & 1.27 (1.11 to 1.44$)$ & $<0.001 \ddagger$ \\
\hline Control & 1.00 & & 1.00 & \\
\hline \multicolumn{5}{|c|}{ Age $\geq 60$ years, men $(n=16920)$} \\
\hline Nephrolithiasis & 1.23 (1.09 to 1.38$)$ & $0.001 \ddagger$ & 1.22 (1.08 to 1.37$)$ & $0.001 \ddagger$ \\
\hline Control & 1.00 & & 1.00 & \\
\hline
\end{tabular}

*Models stratified by age, sex, income and region of residence.

†Adjusted for obesity, smoking, alcohol consumption, fasting blood glucose, total cholesterol and Charlson Comorbidity Index scores.

$\ddagger$ Cox proportional hazards regression model, significance at $\mathrm{p}<0.05$. 
Table 3 Crude and adjusted HRs (95\% Cls) for ischaemic heart disease in the nephrolithiasis and control groups

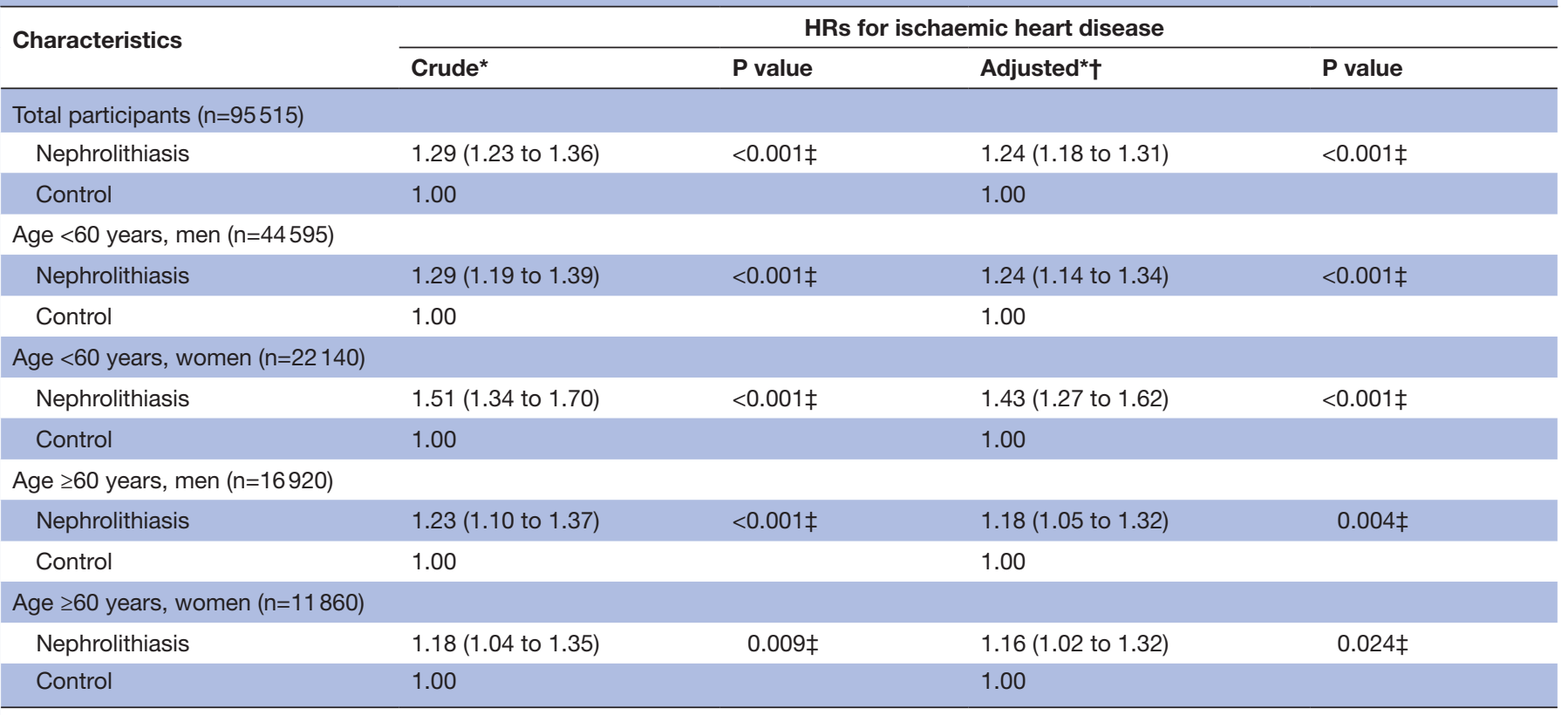

*Models stratified by age, sex, income and region of residence.

†Adjusted for obesity, smoking, alcohol consumption, fasting blood glucose, total cholesterol and Charlson Comorbidity Index scores.

$\ddagger$ Cox proportional hazards regression model, significance at $p<0.05$.

infarction can be explained by the higher rate of urinary tract infection in women than in men, which makes the female population vulnerable to systemic inflammation and atherosclerotic changes. ${ }^{27}{ }^{28}$ In this study, the risk of ischaemic heart disease was increased in both male and female patients with nephrolithiasis. Further analyses adjusting for urinary tract infection (ICD-10: N30, 300,301, 302, 303, 304, 308, 309, 340,341 and 342) were conducted, which showed the consistent association of nephrolithiasis with stroke and ischaemic heart disease, except for in the subgroup of women aged $\geq 60$ years, in whom there was no significant association between nephrolithiasis and ischaemic heart disease (online supplemental tables S7-S9). The large sample population, matched control group and adjusted covariates meant that there were sufficient participants in the male subgroups, increasing the statistical power of this study. On the contrary, there was no association between nephrolithiasis and stroke in the $\geq 60$-year-old male group in this study. The relatively small size of this subgroup could have led to the non-significant association in this group. In addition, the decreased rate of urinary tract infection and increased health-related quality of life in older men could attenuate the impact of nephrolithiasis on stroke. $^{27} 29$

The longitudinal follow-up study design with a control group matched for demographic and socioeconomic factors enabled the elucidation of the previously mixed results on the causal association between nephrolithiasis and cardiovascular diseases. Medical histories and lifestyle factors were comprehensively adjusted for using the CCI and a survey of obesity, smoking and alcohol consumption. In addition, subgroup analyses were performed stratified by obesity, smoking and alcohol consumption. However, primarily because this study used medical claims data, a few limitations should be considered when interpreting the present results. Because these data were based on medical claim codes, laboratory findings, such as serum creatinine, bicarbonate, haemoglobin Alc and serum calcium levels, could not be obtained. In addition, subclinical or untreated patients might have been missed in the present results. The types of nephrolithiasis were not specified; thus, nephrolithiasis was heterogeneous in this study. To estimate the differences according to the severity of nephrolithiasis, subgroup analyses were conducted comparing patients with mild to moderate and severe nephrolithiasis. In addition, this study used a Korean national cohort; therefore, there could be ethnic differences in the association between nephrolithiasis and cardiovascular diseases. ${ }^{30}$

\section{CONCLUSION}

Nephrolithiasis was associated with increased risks of stroke and ischaemic heart disease in men and women aged $\geq 40$ years. Mild to moderate and severe nephrolithiasis were related to elevated risks of stroke and ischaemic heart disease. This relationship was consistent after considering comorbidities and lifestyle factors, including obesity, smoking and alcohol consumption.

\section{Author affiliations}

${ }^{1}$ Otorhinolaryngology-Head \& Neck Surgery, CHA University, Seongnam, The Republic of Korea

${ }^{2}$ Department of Urology, Hallym University College of Medicine, Anyang-si, Gyeonggi-do, The Republic of Korea

${ }^{3}$ Hallym Data Science Laboratory, Hallym University College of Medicine, Chuncheon, Gangwon, The Republic of Korea

${ }^{4}$ Otorhinolaryngology-Head \& Neck Surgery, Hallym University, Anyang-si, The Republic of Korea 
Contributors HGC designed the study; WJB, CM and HGC analysed the data; SYK and WJB drafted and revised the paper; all authors approved the final version of the manuscript.

Funding This work was supported in part by a research grant (NRF-2018R1D1A1A02085328) from the National Research Foundation (NRF) of Korea and the Hallym University Research Fund (HURF-2018-53).

Competing interests None declared.

Patient consent for publication Not required.

Ethics approval The ethics committee of Hallym University (2017-1102) approved this study. The need to obtain written informed consent was waived by the institutional review board. All analyses adhered to the guidelines and regulations of the ethics committee of Hallym University.

Provenance and peer review Not commissioned; externally peer reviewed.

Data availability statement Data are available in a public, open access repository. Release of the data by the researcher is not allowed legally. All data are available from the database of National Health Insurance Sharing Service (NHISS) (https:// nhiss.nhis.or.kr/). NHISS allows all of these data for any researcher who promises to follow the research ethics with some cost. If one wants to access the data of this article, one can download it from the website after promising to follow the research ethics requirements.

Supplemental material This content has been supplied by the author(s). It has not been vetted by BMJ Publishing Group Limited (BMJ) and may not have been peer-reviewed. Any opinions or recommendations discussed are solely those of the author(s) and are not endorsed by BMJ. BMJ disclaims all liability and responsibility arising from any reliance placed on the content. Where the content includes any translated material, BMJ does not warrant the accuracy and reliability of the translations (including but not limited to local regulations, clinical guidelines, terminology, drug names and drug dosages), and is not responsible for any error and/or omissions arising from translation and adaptation or otherwise.

Open access This is an open access article distributed in accordance with the Creative Commons Attribution Non Commercial (CC BY-NC 4.0) license, which permits others to distribute, remix, adapt, build upon this work non-commercially, and license their derivative works on different terms, provided the original work is properly cited, appropriate credit is given, any changes made indicated, and the use is non-commercial. See: http://creativecommons.org/licenses/by-nc/4.0/.

ORCID iD

Hyo Geun Choi http://orcid.org/0000-0003-1655-9549

\section{REFERENCES}

1 Scales CD, Smith AC, Hanley JM, et al. Prevalence of kidney stones in the United States. Eur Urol 2012;62:160-5.

2 Kim Hhoe, Jo MK, Kwak C, et al. Prevalence and epidemiologic characteristics of urolithiasis in Seoul, Korea. Urology 2002;59:517-21.

3 Shoag J, Tasian GE, Goldfarb DS, et al. The new epidemiology of nephrolithiasis. Adv Chronic Kidney Dis 2015;22:273-8.

4 Romero V, Akpinar H, Assimos DG. Kidney stones: a global picture of prevalence, incidence, and associated risk factors. Rev Urol 2010;12:e86-96.

5 Taylor EN, Stampfer MJ, Curhan GC. Obesity, weight gain, and the risk of kidney stones. JAMA 2005;293:455-62.

6 Rendina D, De Filippo G, D'Elia L, et al. Metabolic syndrome and nephrolithiasis: a systematic review and meta-analysis of the scientific evidence. J Nephrol 2014;27:371-6.

7 Sakhaee K. Nephrolithiasis as a systemic disorder. Curr Opin Nephrol Hypertens 2008;17:304-9.

8 Obligado SH, Goldfarb DS. The association of nephrolithiasis with hypertension and obesity: a review. Am J Hypertens 2008;21:257-64.
9 Kim SY, Song CM, Bang W, et al. Nephrolithiasis predicts ischemic stroke: a longitudinal follow-up study using a national sample cohort. Int J Med Sci 2019;16:1050-6.

10 Liu Y, Li S, Zeng Z, et al. Kidney stones and cardiovascular risk: a meta-analysis of cohort studies. Am J Kidney Dis 2014;64:402-10.

11 Peng J-P, Zheng H. Kidney stones may increase the risk of coronary heart disease and stroke: a PRISMA-Compliant meta-analysis. Medicine 2017;96:e7898.

12 Yoshimura E, Sawada SS, Lee I-M, et al. Body mass index and kidney stones: a cohort study of Japanese men. J Epidemiol 2016;26:131-6.

13 Fazlioglu A, Salman Y, Tandogdu Z, et al. The effect of smoking on spontaneous passage of distal ureteral stones. BMC Urol 2014;14:27.

14 Kim SY, Min C, Oh DJ, et al. Tobacco smoking and alcohol consumption are related to benign parotid tumor: a nested casecontrol study using a national health screening cohort. Clin Exp Otorhinolaryngol 2019;12:412-9.

$15 \mathrm{Kim}$ SY, Song CM, Lim H, et al. Bidirectional association between gallstones and renal stones: two longitudinal follow-up studies using a national sample cohort. Sci Rep 2019;9:2620.

16 Ilic M, Grujicic Sipetic S, Ristic B, et al. Myocardial infarction and alcohol consumption: a case-control study. PLoS One 2018;13:e0198129.

17 Schröder H, Masabeu A, Marti MJ, et al. Myocardial infarction and alcohol consumption: a population-based case-control study. Nutr Metab Cardiovasc Dis 2007;17:609-15.

18 Pacific WHOROftW. The Asia-Pacific perspective : redefining obesity and its treatment. Sydney: Health Communications Australia, 2000.

19 Quan H, Li B, Couris CM, et al. Updating and validating the Charlson comorbidity index and score for risk adjustment in hospital discharge Abstracts using data from 6 countries. Am J Epidemiol 2011;173:676-82.

20 Quan H, Sundararajan V, Halfon P, et al. Coding algorithms for defining comorbidities in ICD-9-CM and ICD-10 administrative data. Med Care 2005;43:1130-9.

$21 \mathrm{Kim}$ SY, Kim H-J, Lim H, et al. Bidirectional association between gastroesophageal reflux disease and depression: two different nested case-control studies using a national sample cohort. Sci Rep 2018;8:11748.

22 Kim SY, Lim J-S, Kong IG, et al. Hearing impairment and the risk of neurodegenerative dementia: a longitudinal follow-up study using a national sample cohort. Sci Rep 2018;8:15266.

23 Schlieper G, Westenfeld R, Brandenburg V, et al. Inhibitors of calcification in blood and urine. Semin Dial 2007;20:113-21.

24 Jeong IG, Kang T, Bang JK, et al. Association between metabolic syndrome and the presence of kidney stones in a screened population. Am J Kidney Dis 2011;58:383-8.

25 Iba A, Kohjimoto Y, Mori T, et al. Insulin resistance increases the risk of urinary stone formation in a rat model of metabolic syndrome. BJU Int 2010;106:1550-4.

26 Kohjimoto Y, Sasaki Y, Iguchi M, et al. Association of metabolic syndrome traits and severity of kidney stones: results from a nationwide survey on urolithiasis in Japan. Am J Kidney Dis 2013;61:923-9.

27 Dai H, Guang X, Xiao Z. Increased cardiovascular risk in women with kidney stones: urinary tract infection should be considered. Am J Kidney Dis 2015;65:170.

28 Liu Y, Li S, Qin X. In reply to 'increased cardiovascular risk in women with kidney stones: urinary tract infection should be considered'. Am J Kidney Dis 2015;65:170-1.

29 Stern KL, Gao T, Antonelli JA, et al. Association of patient age and gender with kidney stone related quality of life. J Urol 2019;202:309-13.

30 Glover LM, Bass MA, Carithers T, et al. Association of kidney stones with atherosclerotic cardiovascular disease among adults in the United States: considerations by race-ethnicity. Physiol Behav 2016;157:63-6. 